\title{
Serial composition of quantum coin flipping and bounds on cheat detection for bit commitment
}

\author{
Carlos Mochon* \\ Institute for Quantum Information, California Institute of Technology, Pasadena, California 91125, USA
}

(Received 4 April 2004; published 17 September 2004)

\begin{abstract}
Quantum protocols for coin flipping can be composed in series in such a way that a cheating party gains no extra advantage from using entanglement between different rounds. This composition principle applies to coin-flipping protocols with cheat sensitivity as well, and is used to derive two results: There are no quantum strong coin-flipping protocols with cheat sensitivity that is linear in the bias (or bit-commitment protocols with linear cheat detection) because these can be composed to produce strong coin flipping with arbitrarily small bias. On the other hand, it appears that quadratic cheat detection cannot be composed in series to obtain even weak coin flipping with arbitrarily small bias.
\end{abstract}

DOI: 10.1103/PhysRevA.70.032312

PACS number(s): 03.67.Lx

\section{INTRODUCTION}

Coin flipping is the cryptographic problem where two mutually distrustful parties try to agree on a random bit. This is to be accomplished by sending sequential messages over a communication channel.

The goal is that, if both players are honest and follow the prescribed protocol, they both output the same bit, which is uniformly random. The restriction is that, if either party is dishonest, then the bit output by the honest player must still be approximately random. The figure of merit for a coinflipping protocol is the bias, defined as the maximum increase in the probabilities of the honest player's outputs that a cheating party can achieve.

There is a weaker version of the above problem, where one party wants to obtain outcome zero, and the other party outcome one. This corresponds more closely to the colloquial idea of coin flipping. In this case, the protocol need only prevent a cheater from biasing the coin in favor of his desired outcome. The two versions of the problem are known, respectively, as strong and weak coin flipping.

In the classical setting the problem is impossible without some additional restrictions on the computational power available to each party. Nonetheless, in the quantum setting, where the parties can process quantum information and communicate over a quantum channel, the goal can be partially achieved.

Ambainis [1] and Spekkens and Rudolph [2] have constructed strong coin-flipping protocols with a bias $\epsilon=\frac{1}{4}$. However, it was proven by Kitaev [3] (and summarized in Ref. [4]), that all quantum protocols for strong coin flipping must have a bias of at least $\epsilon=\frac{1}{\sqrt{2}}-\frac{1}{2} \simeq 0.21$.

Quantum weak coin flipping is a little more promising. The best known protocol is by Spekkens and Rudolph [5] and achieves a bias of $\epsilon=\frac{1}{\sqrt{2}}-\frac{1}{2}$. The only known bound is due to Ambainis [1], and states that the number of rounds must grow at least as $\Omega\left(\log \log \frac{1}{\epsilon}\right)$. In particular, to obtain arbitrarily small bias, we must have protocols with an ever increasing number of rounds.

*Electronic address: carlosm@theory.caltech.edu
Unfortunately, analyzing quantum protocols with a large number of rounds is often difficult. One approach to obtaining a weak coin-flipping protocol with arbitrarily small bias could be to take a quantum coin-flipping protocol with a fixed number of rounds and compose it in series with itself to obtain a better coin-flipping protocol. It is well known that quantum coin protocols compose well in series, and an argument for this is given in Sec. II.

Unfortunately, serially composing standard coin-flipping protocols does not decrease the overall bias [6]. However, quantum mechanics is good at detecting state disturbance and other deviations from a protocol. It is therefore possible to construct coin-flipping protocols with cheat sensitivity, where a dishonest player may be able to cheat by a significant amount, but only at the risk of getting caught by the honest player. Cheat sensitive protocols can produce improved coin-flipping protocols when composed in series under certain conditions.

Quantum protocols with cheat sensitivity have been constructed by Spekkens and Rudolph [5] where players cheating by large amounts will get caught, but at least one party can bias the coin by a small amount without being detected. Aharonov et al. [7] have a protocol with quadratic cheat detection on one side, but no cheat detection on the other side. Hardy and Kent [8] devised a protocol where neither player may cheat by any amount without risking detection, but the functional form of the cheat detection was not determined, though it is known to be quadratic or worse. Functional forms of cheat detection were also discussed in Ref. [9].

In the present paper, we will analyze the serial composition of cheat sensitive coin-flipping protocols. We shall treat the cheat sensitive protocols as oracles or black boxes, with a cheat sensitivity that is given by a function of the target bias. This will lead to our two main results.

In Sec. III we show that quadratic cheat detection protocols, where the probability of getting caught is proportional to the square of the bias, are a fixed point of serial composition, at least to leading order. This means that no matter how many times the protocol is composed with itself, the amount of cheat detection remains approximately the same. Because most known cheat sensitive protocols are quadratic 
or worse, this result is evidence that serial composition may not be useful to obtain weak coin flipping.

In Sec. IV we show that linear cheat detection cannot exist for strong coin flipping. This is done by composing the linear cheat detection to obtain a strong coin-flipping protocol with arbitrarily small bias, in violation of Kitaev's lower bound. Note that the second result only uses serial composition as a tool for the proof, and the result holds for all cheat sensitive strong coin-flipping protocols.

The result of Sec. IV also applies to bit commitment, which is a cryptographic protocol related to coin flipping. Coin flipping can be constructed out of bit commitment as follows: first Alice commits a bit to Bob, then Bob announces a random bit, then Alice reveals her bit and the coin outcome is the XOR of the two bits. Cheat detection as a function of $\epsilon$ can be defined in a way similar to Ref. [7]. For Alice, $\epsilon$ is the amount by which she can change the probabilities associated with the committed bit, whereas for Bob, $\epsilon$ is the additional probability of guessing Alice's committed bit correctly. Because linear cheat detection in bit commitment can be used to produce a strong coin-flipping protocol with linear cheat detection, it is also ruled out as a possible quantum protocol.

\section{SERIAL COMPOSITION OF QUANTUM COIN PROTOCOLS}

Protocols for coin flipping can be naturally composed in series to obtain new coin-flipping protocols. What is surprising at first is that quantum protocols for coin flipping compose serially in such a way that a cheating party does not get any unexpected advantage by using entanglement. We shall prove this below, but let us first define carefully what we mean by unexpected advantage.

Let $\mathcal{P}$ be any quantum coin-flipping protocol. At the end of the protocol each player will output one of $\{0,1, C\}$ where the last entry denotes the output when one player catches the other player cheating. Let us assume that Alice is honest and Bob is cheating. For each cheating strategy employed by $\mathrm{Bob}$, there will be a triple of probabilities $\left(P_{0}, P_{1}, P_{C}\right)$, one for each of Alice's possible outputs. Let $\Omega_{A}(\mathcal{P})$ be the set of all such attainable triples. Clearly $(1 / 2,1 / 2,0) \in \Omega_{A}(\mathcal{P})$ since Bob can always play honestly. If the protocol does not allow Bob to fully bias toward 1 then $(0,1,0) \notin \Omega_{A}(\mathcal{P})$. Some protocols may not have any cheat detection, in which case $P_{C}$ will always be zero. For honest Bob and cheating Alice there is a similarly defined $\Omega_{B}(\mathcal{P})$.

Assume we take the protocol $\mathcal{P}$ and run it many times in series. We are interested in proving that a cheating player, say Bob, does not obtain any extra cheating power by using entanglement between different rounds. That is, that for every round $j$, independently of previous outcomes and strategies used by Bob, any strategy that Bob employs will make Alice output based on a triple of probabilities in $\Omega_{A}(\mathcal{P})$.

Clearly Bob can vary his strategy in each coin-flip round, and even base his strategy on the outcomes of the previous coin flips. However, when flipping $N$ coins in series, Bob cannot obtain an outcome of all ones with a probability greater than $\left(P_{1, \max }\right)^{N}$, where $P_{1, \text { max }}$ is the maximum value of $P_{1}$ over any triple in $\Omega_{A}(\mathcal{P})$.

The reason that quantum coin flipping can be serially composed stems from the following conditions which are always imposed on coin-flipping protocols.

(1) There is always at least one honest party.

(2) The details of the protocol, which can be described in terms of fixed unitaries acting on a fixed initial state, are known to all parties.

(3) The protocol begins in an unentangled state, a condition which can be enforced by the honest party.

The first condition arises because no constraint is imposed on the case when both parties are cheating, and therefore the case never needs consideration. The third condition is always imposed to avoid trivial protocols, since establishing correlations is the goal of a coin-flipping procedure.

The proof of composition is fairly simple. At the beginning of the $k$ th round, the cheater will be unentangled from the honest player. The honest player has erased her Hilbert space and reset it to the initial state of the protocol. All that the cheating player has left over from the previous rounds is some quantum state in his Hilbert space. However, because the honest protocol is public, the cheating player knows exactly the state of his Hilbert space (which may be a mixed state, caused by the honest player erasing her Hilbert space).

Now assume that he could, with the help of this state, force the honest player (say Alice) to output with probabilities not in $\Omega_{A}(\mathcal{P})$. Then with the same state, he could obtain the same results in the first round or even when protocol $\mathcal{P}$ is used in a one-shot run, contradicting the definition of $\Omega_{A}(\mathcal{P})$. To put it another way, Bob can simulate in his private Hilbert space the first $k-1$ rounds and start playing the first round from that point, but this clearly can give him no extra advantage.

The conclusion of this section is that, given a quantum protocol for coin flipping, we may treat the protocol as a black box when composing it in series with itself (or even with other coin-flipping protocols) without worrying about entanglement between rounds. We shall use this fact to derive two interesting results.

\section{QUADRATIC CHEAT DETECTION IS A FIXED POINT OF COIN FLIPPING}

Because quantum coin flipping composes in series, it is tempting to try to use a classical game layer on top of a known quantum coin-flipping protocol in order to reduce its maximum bias. That is, we wish to construct a two player classical game that uses the quantum coin as a black box. The game could be, for instance, flipping a coin $N$ times, and the party that wins a majority of coin tosses wins the game.

The ideal goal for this process would be to produce a weak coin-flipping protocol with arbitrarily small bias. While it is known that games built out of standard coin-flipping protocols can never reduce the maximum bias, the situation is different when cheat detection is available. Especially in the case of weak coin flipping, where an honest player may declare himself the winner if he detects the other party cheating, there are certain black-box protocols that can be used to 


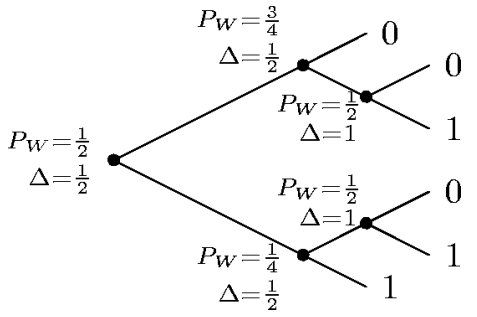

FIG. 1. "Best two out of three" game tree. Binary nodes are labeled by the probability of winning for an honest player trying to obtain outcome 0 .

produce arbitrarily small bias. The question is how much cheat detection is needed in order for successive compositions to improve a coin-flipping protocol?

We will be interested in protocols with symmetric, monomial cheat detection. Let $\mathcal{P}$ be a protocol where both parties have equal cheating opportunities $\left(\Omega_{A}(\mathcal{P})=\Omega_{B}(\mathcal{P}) \equiv \Omega(\mathcal{P})\right)$ and such that all probabilities $\left(P_{0}, P_{1}, P_{C}\right) \in \Omega(\mathcal{P})$ have the form

$$
\begin{gathered}
P_{0}=\left(1-P_{C}\right)\left(\frac{1}{2}+\epsilon\right), \\
P_{1}=\left(1-P_{C}\right)\left(\frac{1}{2}-\epsilon\right), \\
P_{C} \geqslant a|\epsilon|^{b},
\end{gathered}
$$

which can be viewed as a function of a parameter $\epsilon$ that is controlled by the cheating party. The constants $a>0$ and $b$ $\geqslant 0$ denote the amount of cheat detection. Strictly speaking, $P_{C}$ should also be considered a second parameter that can be controlled by the cheater, as he can always use a less optimal cheating strategy. In practice, a cheater will always minimize $P_{C}$ for a given bias, and therefore we may assume equality in Eq. (3). We shall call the case $b=1$ linear cheat detection, and the case $b=2$ quadratic cheat detection.

When building games out of cheat sensitive coin flips, the outcome of the entire game will be "cheating" if in any individual coin flip a cheating outcome was obtained. To leading order in epsilon, the composite game will have a cheat sensitivity of the same form, with the same coefficient $b$, but in general a different $a$. When $b$ is small, successive compositions increase $a$ thereby producing a more cheat sensitive protocol, whereas in the large $b$ regime composition has the opposite effect. We intend to prove in this section that $b=2$ is a fixed point of coin flipping. That is, for all possible games that use a coin with quadratic cheat detection as a black box, the resulting protocol has exactly the same amount of cheat detection to leading order in epsilon.

We begin by describing the set of all possible games that employ a cheat sensitive coin flip as a black box. These can be put in correspondence with the set of binary trees, where the leaf nodes are labeled by either zero or one. For instance, the tree corresponding to the "Best two out of three" game is depicted in Fig. 1. Each binary node corresponds to a coin flip, with up corresponding to the outcome zero and down to the outcome one. The leaves are end points of the game and their labels correspond to the final game outcome at that node.

Other examples of games that produce coin flips include "first outcome that is repeated $N$ times" and "first outcome to occur a total of $N$ times more than the other," both of which correspond to trees of infinite length. Allowing for trees of infinite length also accounts for all games that have a tie outcome, after which the game is restarted. For trees of infinite length we impose the additional constraint that when playing honestly, the probability of never reaching a leaf node is zero.

Let $x$ be a variable that ranges over the binary nodes of the tree. A general cheating strategy is a function $\epsilon(x)$, which assigns a bias to each node where a coin flip takes place. We also define $p_{\epsilon}(x)$ as the probability of arriving at node $x$ given a cheating strategy $\epsilon(x)$. In defining this quantity, we assume that if the cheater is caught at a given node, the game stops, thereby reducing the probability of arriving at the child nodes. In terms of these quantities we can write the total probability of getting caught:

$$
P_{C, t o t}=\sum_{x} a p_{\epsilon}(x)|\epsilon(x)|^{b} .
$$

To leading order in $\epsilon(x)$, we can replace $p_{\epsilon}(x)$ with the probability of arriving at the node in the honest protocol. This can be written as $2^{-D(x)}$ where $D(x)$ is the depth of the node $x$, with the root node having depth zero. The formula becomes

$$
P_{C, t o t}=\sum_{x} a 2^{-D(x)}|\epsilon(x)|^{b} .
$$

To expand the total bias to leading order in epsilon, we only need to keep track of terms that are linear in $\epsilon(x)$, and can therefore disregard the multiplicative factor $1-P_{C}$ in the probabilities for obtaining zero or one. Of course, we are assuming $b>1$ at this point, and will soon concentrate on $b=2$. The probability of the cheater obtaining his desired outcome, and winning the game, satisfies a simple recursion relation:

$$
P_{W}(x)=\frac{1}{2}\left[P_{W}\left(x^{\uparrow}\right)+P_{W}\left(x^{\downarrow}\right)\right]+\epsilon(x)\left[P_{W}\left(x^{\uparrow}\right)-P_{W}\left(x^{\downarrow}\right)\right],
$$

where $P_{W}(x)$ is the probability of winning having arrived at node $x$, and $x^{\uparrow}, x^{\downarrow}$ are the two children of node $x$. At the root node the probability of winning is

$$
P_{W, t o t}=\frac{1}{2}+\sum_{x} 2^{-D(x)} \Delta(x) \epsilon(x),
$$

where $\Delta(x)=P_{W}\left(x^{\uparrow}\right)-P_{W}\left(x^{\downarrow}\right)$, which can be computed at this point from the honest probability of winning.

The total bias for the game is given to leading order by $\left|\epsilon_{t o t}\right|=P_{W}-1 / 2$, where we have excluded cases where the cheating benefits the honest player. For a given total bias, the cheater will choose $\epsilon(x)$ to minimize the probability of get- 
ting caught. The calculation to minimize $P_{C, t o t}$ under the constraint of fixed $\epsilon_{t o t}$ can easily be done using a Lagrange multiplier to obtain the result:

$$
\epsilon(x)=\operatorname{sgn}[\lambda \Delta(x)]\left|\frac{\lambda \Delta(x)}{a b}\right|^{1 /(b-1)},
$$

where $\lambda$ is the Lagrange multiplier. We have allowed $\epsilon(x)$ to range over the reals, but will show below that for quadratic cheat detection the optimal solution satisfies the requirement $|\epsilon(x)| \leqslant 1 / 2$.

To eliminate the Lagrange multiplier, we can substitute the expression for $\epsilon_{t o t}$ into the expression for $P_{C, t o t}$ to obtain

$$
P_{C, t o t}=a_{\text {new }}\left|\epsilon_{t o t}\right|^{b},
$$

where

$$
a_{\text {new }}=a\left(\sum_{x} 2^{-D(x)}|\Delta(x)|^{b /(b-1)}\right)^{1-b} .
$$

For the case $b=2$, the following lemma shows that $a_{\text {new }}$ $=a$ for all game trees:

Lemma: For all game trees as described above, we have the equality

$$
\sum_{x} 2^{-D(x)} \Delta(x)^{2}=1
$$

The lemma can be proven using a simple combinatorial argument that is presented for the interested reader in the Appendix.

From Eq. (8) we see that for $b=2$, the amount of cheating at node $x$ is proportional to $\Delta(x)$. The constant of proportionality, as well as the Lagrange multiplier, are fixed by Eq. (7). After eliminating a factor of 1 using the above lemma we obtain that the optimal strategy is $\epsilon(x)=\left|\epsilon_{t o t}\right| \Delta(x)$. This satisfies the intuition that the cheater will choose a larger bias on coin flips that are more consequential. Furthermore, because $|\Delta(x)| \leqslant 1$, we have shown that the optimal strategy is achievable (i.e., $|\epsilon(x)| \leqslant 1 / 2$ for every $x$ ) whenever $\left|\epsilon_{t o t}\right|$ $\leqslant 1 / 2$.

For other values of $b$ near 2, we can consider the derivative of $a_{\text {new }}$ with respect to $b$. For any fixed graph, this derivative is negative. The conclusion is that for every graph, serial composition of $b<2$ cheat detection leads to improvement to lowest order, whereas in the $b>2$ case the cheat sensitivity worsens.

The above results complete our argument that quadratic cheat detection is a fixed point for coin flipping. The argument is only valid in the regime where all biases, including the total bias, are small. Unfortunately, because a protocol producing weak coin flipping with arbitrarily small bias would have to employ arbitrarily large trees, it is not sufficient to simply take the small bias limit on a fixed tree.

In essence, associated with each weak coin-flipping protocol there is a function $P_{C}(\epsilon)$ indicating the minimum probability with which a party will get caught cheating if they try to bias the coin by $\epsilon$. Composing this protocol in series one obtains a new protocol with function $P_{C, t o t}\left(\epsilon_{t o t}\right)$. That is, serial composition with a given game tree induces a map from the set of functions $P_{C}(\epsilon)$ to itself. We have shown that, independent of the tree, if the original function behaves as $a \epsilon^{2}$ for small $\epsilon$, then so will its image.

The fact that the coefficient of the quadratic term remains fixed under the map induced by every game tree is a peculiar and interesting feature. It is indicative that serial composition of quadratic cheat detection may not be useful for producing weak coin flipping with arbitrarily small bias. However, further research in this direction is needed in order to conclusively settle the issue.

\section{NO-GO THEOREM FOR LINEAR CHEAT DETECTION}

In this section we shall switch gears and focus on serial composition of linear cheat detection protocols. We shall prove that linear cheat detection can be serially composed to produce not only weak coin flipping but also strong coin flipping with arbitrarily small bias. Because of Kitaev's bound (discussed in Ref. [4]), the result in this section proves that a strong coin-flipping protocol with linear cheat detection cannot exist.

The result of this section only applies to strong coinflipping schemes with cheat sensitivity as described in Eq. (3). An alternative not covered by the proof in this section is the case where Alice can force outcome 1 (and Bob can force outcome 0) without getting caught, which corresponds to cheat sensitive weak coin flipping. Because weak cheat sensitivity can be simulated by strong cheat sensitivity, the result of the previous section applies to weak cheat sensitivity as well. However, the opposite is not true, and the results of the present section do not apply to linear cheat sensitivity in weak coin flipping.

Since strong coin flipping can be constructed out of bit commitment, the result of this section can also be applied to cheat sensitive bit-commitment protocols. In fact, the only known coin-flipping protocol, where neither side can cheat by a finite amount without getting caught, is described in Ref. [8], and is a strong coin-flipping protocol that is constructed out of bit commitment.

For the proof of the above statements, we assume the existence of a quantum protocol $\mathcal{P}$ described by Eq. (3) with $b=1$ and some nonzero $a$. We shall describe a game that uses $\mathcal{P}$ as a black box, which achieves strong coin flipping with bias that becomes arbitrarily small in the limit of a game parameter $N \rightarrow \infty$. Though, for the purposes of comparing against Kitaev's bound, it is sufficient to allow honest players to output "cheat" at the end, we will construct the game so that the honest player always outputs one of the outcomes zero or one.

The game is the random walk on a one-dimensional line, starting from the origin, using the coin provided by $\mathcal{P}$. The game ends with the first arrival at one of the two sites $\pm N$, with the right end corresponding to the outcome zero, and the left end to one.

If one party detects cheating, they will continue the game using a private fair coin, and output according to the outcome of the game. Let $z \in\{-N, \ldots, N\}$ be a variable that runs along the line. If cheating occurs when the game is at $z$, then the honest players can simply output using $P_{0}=(N+z) / 2 N$ and $P_{1}=(N-z) / 2 N$. In essence, the only deterrent to the 
cheater is that he may be able to cheat more effectively in a future round. Note that the honest party cannot just flip a balanced coin after detecting cheating because in this case the cheating party would only cheat when he is about to lose.

Because the honest player keeps no state beyond the current location along the line, $z$, there is an optimal cheating strategy where the bias only depends on $z$. That is, we only need to consider functions $\epsilon(z)$ when maximizing over cheating strategies.

We assume that the cheating player is trying to bias toward zero (i.e., the right side). We can then define the function $W_{\epsilon}(z)$ to be the probability of winning starting from node $z$, using biases $\epsilon(z)$. The function is similar to $P_{W}$ defined in the previous section, except that we are now using large biases, which cannot be ignored in calculating the probability of winning.

The function has the constraints $W_{\epsilon}(N)=1, W_{\epsilon}(-N)=0$ and

$$
\begin{aligned}
W_{\epsilon}(z)= & P_{0}[\epsilon(z)] W(z+1)+P_{1}[\epsilon(z)] W(z-1) \\
& +P_{C}[\epsilon(z)] \frac{N+z}{2 N} .
\end{aligned}
$$

Clearly, for optimal strategies, $W_{\epsilon}(z) \geqslant(N+z) / 2 N$ for all $z$ because the cheating party can always play honestly.

The value of $W_{\epsilon}(0)$ is simply the probability of winning the entire game. The cheater will chose $\epsilon(z)$ in order to maximize $W_{\epsilon}(0)$. We shall prove that $\max \left[W_{\epsilon}(0)-1 / 2\right] \rightarrow 0$ as $N \rightarrow \infty$.

The analysis is made easier by using a modified black box protocol $\mathcal{P}^{\prime}$ with achievable probabilities of the form

$$
\begin{gathered}
P_{0}=\frac{1}{2}+\epsilon, \\
P_{1}=\frac{1}{2}-(1+a) \epsilon, \\
P_{C}=a \epsilon,
\end{gathered}
$$

for $0 \leqslant \epsilon \leqslant \epsilon_{\max }$ where $\epsilon_{\max }=1 /(2+2 a)$. For every $\epsilon$, protocol $\mathcal{P}^{\prime}$ gives the cheater a slightly higher probability of obtaining the desired outcome than with protocol $\mathcal{P}$. Therefore, any bounds on cheating obtained using $\mathcal{P}^{\prime}$ as a black box will apply when using $\mathcal{P}$ instead.

Consider using $\mathcal{P}^{\prime}$ and varying independently each of the nodes of the complete game tree. The bias $\epsilon(x)$ of each node enters linearly into $W_{\epsilon}(0)$, which can be maximized by letting the biases take only the boundary values of 0 or $\epsilon_{\max }$. As discussed above, the optimal biases will depend only on the corresponding value of $z$, and therefore we need only consider functions $\epsilon(z)$ which take values in $\left\{0, \boldsymbol{\epsilon}_{\max }\right\}$.

The maximization is now easy to analyze. Define $\delta(z)$ $=W_{\epsilon}(z)-(N+z) / 2 N$, which is the extra probability of winning that the cheater is getting at position $z$. We shall prove below that

$$
\delta(z+1) \leqslant \frac{2+a}{2 a N} \quad \Rightarrow \quad \delta(z) \leqslant \frac{2+a}{2 a N} .
$$

If $\epsilon(z)=\epsilon_{\max }$ the above statement is true because Eq. (12) states that

$$
\begin{aligned}
\delta(z) & =\left(\frac{1}{2}+\epsilon_{\max }\right)\left(\delta(z+1)+\frac{1}{2 N}\right) \\
& =\frac{2+a}{2+2 a}\left(\delta(z+1)-\frac{2+a}{2 a N}\right)+\frac{2+a}{2 a N} .
\end{aligned}
$$

On the other hand, if $\epsilon(z)=0$ then Eq. (12) states that

$$
\delta(z+1)-\delta(z)=\delta(z)-\delta(z-1),
$$

that is, $\delta$ has a constant slope around $z$. If for all $z^{\prime}<z$ we have $\epsilon\left(z^{\prime}\right)=0$, then the slope must be constant through this entire region. Since $\delta(-N)=0$, the slope can only be negative (i.e., increasing toward the left) if $\delta(z)<0$, which proves Eq. (16) for this case. Otherwise, let $z^{\prime}<z$ be the largest integer such that $\epsilon\left(z^{\prime}\right)=\epsilon_{\max }$. Again the slope must be constant from $z^{\prime}$, in which case, by Eq. (18) the slope can only be negative if $\delta\left(z^{\prime}\right)<(2+a) / 2 a N$ which implies $\delta(z)<(2+a) / 2 a N$.

Having proven Eq. (16), and using the initial case $\delta(N)$ $=0$, we have shown

$$
\epsilon_{t o t} \equiv W_{\epsilon}(0)-\frac{1}{2}=\delta(0) \leqslant \frac{2+a}{2 a N},
$$

which can be made arbitrarily small by taking the limit $N$ $\rightarrow \infty$.

The game described in this section shows that a strong coin-flipping protocol with linear cheat detection can be serially composed to obtain a strong coin-flipping protocol with arbitrarily small bias. The conclusion is that quantum strong coin-flipping protocols with linear cheat detection cannot exist.

\section{CONCLUSIONS}

Using serial composition of coin flipping we have established an upper bound on the amount of cheat detection possible in quantum protocols for coin flipping and bit commitment. We have also presented evidence that serially composing quadratic cheat sensitive protocols does not lead to an improvement in the amount of cheat detection. We speculate that quadratic or worse cheat sensitivity $(b \geqslant 2)$ cannot be composed in series to obtain weak coin flipping with arbitrarily small bias. We also speculate that cheat detection better than quadratic $(b<2)$ does not exist for bit commitment or strong coin flipping, and probably not for weak coin flipping either.

Nevertheless, linear cheat detection in weak coin flipping remains an open, though unlikely, possibility. In fact, by serially composing weak coin flipping with linear cheat detection, it is not hard to show that one can construct a weak coin-flipping protocol with a bias of exactly zero. The apparent contradiction with the result of Lo and Chau [10] is resolved by noting that they only considered protocols with a fixed number of rounds. However, there are protocols where the number of rounds is variable and possibly arbitrarily large (a good classical example is rock-paper-scissors), while still having a zero probability of going on forever. For these 
protocols the measurements cannot be delayed to the final round, and therefore the analysis of Lo and Chau does not apply. These protocols can always be truncated to a finite number of rounds, though, at the cost of allowing an arbitrarily small bias.

Other questions that remain open include: What happens to serial composition of quadratic cheat detection in the large bias regime? What can be said for other functional forms of cheat detection versus bias, including cases where one party may be a able to cheat by a small amount without getting caught? And of course, the main question-whether or not weak coin flipping with arbitrarily small bias can be achieved as a quantum protocol—remains unresolved.

\section{ACKNOWLEDGMENTS}

The author would like to thank John Preskill, Michael Ben-Or, Rob Spekkens, and Ben Toner. This work was supported in part by the National Science Foundation under Grant No. EIA-0086038 and by the U.S. Department of Energy under Grant No. DE-FG03-92-ER40701.

\section{APPENDIX: PROOF OF LEMMA}

Let $\mathcal{T}$ be a binary tree. We will use the variable $x$ to denote a binary node of $\mathcal{T}$ and $y$ to denote a leaf.

Associated with $\mathcal{T}$ there is a function $P_{W}(y)$ on the leaf nodes which takes the values zero or one. The function can be extended to the rest of the nodes by defining $P_{W}(x)$ as the average of the function on its two descendants. In terms of coins, $P_{W}$ is simply the probability of winning when playing honestly starting from the given node. The function corresponds to the coin outcomes on the leaf nodes if the goal is to obtain 1 , and otherwise the coin outcome is $1-P_{W}(y)$. To obtain a fair coin toss, we require that $P_{W}$ equal $1 / 2$ on the root node. Otherwise, the function and the tree are arbitrary.

We also arbitrarily label the two outgoing edges from each binary node as up and down, and define $\Delta(x)=P_{W}\left(x^{\uparrow}\right)$ $-P_{W}\left(x^{\downarrow}\right)$. Finally, let $D(x)$ be the depth of node $x$, with the root node having depth zero.
Lemma: Given a tree $\mathcal{T}$ and function $P_{W}$ as described above, the following equality holds:

$$
\sum_{x} 2^{-D(x)} \Delta(x)^{2}=1
$$

Proof. $\Delta(x)$ is a linear combination of $P_{W}(x)$ on its descendants, which in turn is a linear combination of $P_{W}(y)$ on the leaf nodes. Therefore the left-hand side of the above equation is a quadratic polynomial of $P_{W}(y)$.

Fix a leaf node $y$. The function $P_{W}(y)$ only appears in $\Delta(x)$ if $y$ is a descendant of $x$, in which case it has a coefficient of $\pm 2^{D(x)+1-D(y)}$. The coefficient of $P_{W}(y)^{2}$ in this polynomial is therefore given by

$$
\sum_{i=0}^{D(y)-1} 2^{-i} 2^{2[i+1-D(y)]}=2^{2[1-D(y)]}\left(2^{D(y)}-1\right) .
$$

Fix a second leaf node $y^{\prime} \neq y$. Let $x^{\prime}$ be the unique node that has $y$ as a descendant along one branch and $y^{\prime}$ along the other. The coefficient of $P_{W}(y) P_{W}\left(y^{\prime}\right)$ is

$$
\begin{aligned}
& 2\left[\sum_{i=0}^{D\left(x^{\prime}\right)-1} 2^{-i+2(i+1)-D(y)-D\left(y^{\prime}\right)}\right]-2^{D\left(x^{\prime}\right)-D(y)-D\left(y^{\prime}\right)+3} \\
& \quad=-2^{3-D(y)-D\left(y^{\prime}\right)}
\end{aligned}
$$

where the only negative term is contributed by $\Delta\left(x^{\prime}\right)$. Note the extra factor of 2 accounting for the double occurrence of $P_{W}(y) P_{W}\left(y^{\prime}\right)$.

Combining these results, the left-hand side of Eq. (A1) is

$$
4 \sum_{y} 2^{-D(y)} P_{W}(y)^{2}-4\left[\sum_{y} 2^{-D(y)} P_{W}(y)\right]^{2} .
$$

Note that the factor in brackets is just $P_{W}$ at the root node, which must equal $1 / 2$. The left summand can be simplified by using $P_{W}(y)^{2}=P_{W}(y)$, in which case it can also be written in terms of $P_{W}$ at the root node. We have shown that the left-hand side equals $4(1 / 2)-4(1 / 2)^{2}=1$ as desired.
[1] A. Ambainis, in Proceedings on 33rd Annual ACM Symposium on Theory of Computing (ACM, New York, 2001), pp. 134142.

[2] R. W. Spekkens and T. Rudolph, Phys. Rev. A 65, 012310 (2002).

[3] A. Yu. Kitaev, results presented at QIP 2003 (slides and video available from MSRI).

[4] A. Ambainis, H. Buhrman, Y. Dodis, and H. Roehrig, in Proceedings of 19th Annual Conference on Computational Complexity, Amherst, MA, June 21-24 (IEEE Computer Society, Los Alamitos, CA, 2004), pp. 250-259).
[5] R. W. Spekkens and T. Rudolph, Phys. Rev. Lett. 89, 227901 (2002).

[6] M. Santha and U. V. Vazirani, J. Comput. Syst. Sci. 33, 75 (1986).

[7] D. Aharonov, A. Ta-Shma, U. Vazirani, and A. Yao, in Proceedings of the Thirty Second Annual ACM Symposium on Theory Of Computing (ACM, New York, 2000), pp. 705-724.

[8] L. Hardy and A. Kent, Phys. Rev. Lett. 92, 157901 (2004).

[9] R. W. Spekkens and T. Rudolph, quant-ph/0310060.

[10] H.-K. Lo and H. F. Chau, Physica D 120, 177 (1998). 